\title{
Pouvoir antibactérien des extraits aqueux et hydro- éthanolique du mélange d'écorces de tronc d'Albizia gummifera (J.F. Gmel.) C.A. Sm et Spathodea campanulata P.Beauv
}

\author{
Bayaga Hervé Narcisse1, Guedje Nicole Marie1, Tabi OmgbaYves², Pola Yissibi Emilienne ${ }^{3}$, Njinkio \\ Nono Borgia Legrand ${ }^{2}$, Assong Elombat Danielle Cynthia ${ }^{4}$, Fokunang charles ${ }^{2}$, Ntsama Essomba \\ Claudine ${ }^{1,4}$ \\ 1.Département de Pharmacognosie et Chimie Pharmaceutique, Faculté de Médecine et des Sciences Biomédicales, \\ Université de Yaoundé I, B.P. 1364 Yaoundé, Cameroun \\ 2.Département de Pharmacotoxicologie et Pharmacocinétique, Faculté de Médecine et des Sciences Biomédicales, \\ Université de Yaoundé I, B.P. 1364 Yaoundé, Cameroun \\ 3.Département de Pharmacie Galénique et Législation Pharmaceutique, Faculté de Médecine et des Sciences \\ Biomédicales, Université de Yaoundé I, B.P. 1364 Yaoundé, Cameroun \\ 4.Département de Biologie et Physiologie Animales, Laboratoire de Parasitologie et Ecologie, Faculté des Sciences, \\ Université de Yaoundé I, B.P. 812 Yaoundé
}

Original submitted in on $31^{\text {st }}$ July 2020. Published online at www.m.elewa.org/journals/ on $31^{\text {st }}$ October 2020 https://doi.org/10.35759/JABs.154.5

\section{RESUME}

Objectifs : Ce travail avait pour but de déterminer les grands groupes de métabolites secondaires et évaluer le pouvoir antibactérien in vitro des extraits aqueux et hydro-éthanolique d'une recette traditionnelle à base du mélange d'écorces de tronc d'Albizia gummifera et Spathodea campanulata.

Méthodologie et Résultats : La recette a été préparée selon les indications des tradithérapeutes. Le screening phytochimique des extraits aqueux et hydro-éthanolique a révélé la présence de métabolites secondaires: polyphénoles, flavonoïdes, coumarines, tanins, glycosides cardiaques, bétacyanes, saponosides et alcaloides. La détermination des Concentrations Minimales Inhibitrices (CMI), par la technique de macrodilution en milieu liquide a établi le pouvoir antibactérien des extraits vis-à-vis de Staphylococcus aureus ATCC BAA-977 (CMl comprises entre 0,78 et $6,25 \mathrm{mg} / \mathrm{mL}$ ) et Escherichia coli ATCC 25922 (CMI supérieures à $100 \mathrm{mg} / \mathrm{mL}$ ).

Conclusion et application des résultats : Les résultats obtenus de cette étude suggèrent que les métabolites secondaires présents dans les extraits aqueux et hydro-éthanolique du mélange d'écorces de tronc d'Albizia gummifera et Spathodea campanulata avaient des propriétés antibactériennes sur les deux souches testées, particulièrement sur Staphylococcus aureus ATCC BAA-977. Ces résultats justifient l'usage médicinal de cette recette par les tradithérapeutes dans le traitement des plaies infectées à Akonolinga. Cependant, il serait important de mener des études de toxicité, indispensables à la formulation d'un Médicament Traditionnel Amélioré (MTA) avec pour but de contribuer à la valorisation de notre médecine et pharmacopée traditionnelles.

Mots clés : Composition phytochimique, pouvoir antibactérien, Albizia gummifera, Spathodea campanulata 
Antibacterial effect of aqueous and hydro-ethanolic extracts of the mixture of trunk bark of Albizia gummifera (J.F. Gmel.) C.A. Sm and Spathodea campanulata P. Beauv

\begin{abstract}
Objectives: The objectives of this work was to determine groups of secondary metabolites and to assess in vitro antibacterial activity of aqueous and hydro-ethanolic extracts of a traditional recipe based on the mixture of trunk bark of Albizia gummifera and Spathodea campanulata.

Methodology and Results: The recipe was prepared according to the instructions of traditional healers. The phytochemical screening of the aqueous and hydro-ethanolic extracts of this recipe revealed the presence of several families of chemical compounds like : polyphenols, flavonoïdes, coumarines, tannins, cardiac glycosides, betacyanes, saponosides et alcaloides with antibacterial potential. The determination of the Minimum Inhibitory Concentrations (MIC), by the technique of macro-dilution in liquid medium, made it possible to evaluate the antibacterial activities of the extracts. These different extracts inhibited the visible bacterial growth of Staphylococcus aureus ATCC BAA-977 (MIC between 0.78 and $6.25 \mathrm{mg} / \mathrm{mL}$ ) and Escherichia coli ATCC 25922 (MIC greater than $100 \mathrm{mg} / \mathrm{mL}$ ).

Conclusion and application of results: These results suggest that the aqueous and hydro-ethanolic extracts for the antibacterial activity observed against the two strains of the species tested and particularly against Staphylococcus aureus ATCC BAA-977. Thus, these results would justify the empirical medicinal use of this recipe by traditional therapists in Akonolinga and therefore constitutes a potential candidate for toxicity studies, essential for the formulation of an Improved Traditional Medicine (MTA) in order to contribute to the enhancement of our traditional medicine and pharmacopoeia.
\end{abstract}

Keywords: phytochemical composition, antibacterial power, Albizia gummifera, Spathodea campanulata.

\section{INTODUCTION}

Les maladies infectieuses demeurent une importante préoccupation de santé publique à cause de leur fréquence et de leur gravité surtout dans les pays en voie de développement (Traoré et al., 2012). En effet, d'après un rapport du haut Conseil de la Santé Publique de France en 2011, sur un total de 57 millions de décès annuels à travers le monde, $30 \%$ sont causés par les infections dont $70 \%$ sont d'origine bactérienne. La prise en charge de ces infections consiste à administrer aux patients les antibiotiques dans un but curatif ou préventif. Cependant, l'utilisation anarchique, inadéquate et abusive des antibiotiques en médecine humaine et vétérinaire, a provoqué l'émergence de bactéries multi résistantes pouvant être responsables des échecs thérapeutiques et des coûts élevés des traitements. Pour pallier à cette situation, l'Organisation Mondial de la Santé recommande la recherche de nouvelles molécules médicamenteuses et l'utilisation en combinaison celles déjà existantes (OMS, 2013). Par ailleurs, elle reconnait en la médecine traditionnelle à base de plantes, dont $80 \%$ de la population africaine ont recours, une source sûre de remèdes pour la prise en charge des maladies d'origine microbienne ou non. De nombreuses études ont décrit l'utilisation en médecine traditionnelle de différents organes végétaux de Spathodea campanulata P.Beauv et d'Albizia gummifera (J.F. Gmel.) C.A. Sm. dans le traitement des maladies urinaires, des dermatoses (Ulcère de Buruli, plaies, gale), de l'herpès, des maux de ventre et des diarrhées (Yemoa et al.,2011; Adjet et al., 2016; Pone Kamdem, 2017, Wagh \& Butle, 2018). Au Cameroun, des observations préliminaires, menées auprès de neuf tradipraticiens à Akonolinga (Région du CentreCameroun) par Bayaga et al.,2017 ont permis d'identifier le décocté du mélange des écorces de tronc de ces deux espèces végétales, comme détenteur d'un fort potentiel thérapeutique vis-à-vis des pathologies infectieuses. Rajesh et al., 2010 a démontré que l'extrait éthanolique des fleurs de $S$. campanulata était plus active que celui des feuilles sur les souches de bactéries à Gram+ et à Gram- et attribue cette activité à la présence des flavonoïdes 
et des tannins; Tanayen et al., 2016 a identifié les métabolites secondaires et démontré l'activité antidiabétique de l'extrait méthanolique des écorces de tronc de $S$. campanulata, tandis que Wagh \& Butle,2018 dans un article de revue ont fait la revue du profil chimique et pharmacologique des extraits de différentes parties de $S$. campanulata et ont relevé l'activité antibactérienne de différents extraits d'écorces de cette plante sur de nombreuses souches bactériennes. Plus tard, Wagh et al. ;2019 a démontrer l'activité antihelminthique de l'extrait méthanolique des feuilles de $S$. campanulata sur les

\section{MATÉRIEL ET MÉTHODES}

Matériel végétal : Les écorces de tronc de Spathodea. campanulata P.Beauv et d'Albizia. gummifera (J.F. Gmel.) C.A. Sm ont été récoltées à Akonolinga dans la région du Centre Cameroun. L'authentification des échantillons de chaque plante ont été faites par comparaison avec des spécimens de l'Herbier National du Cameroun à Yaoundé en 2015. L'échantillon de $S$. campanulata P.Beauv a été authentifié en comparaison avec celle du collecteur Nana Pierre 277 du spécimen de la collection d'herbier à la référence numéro 2133. Tandis que l'échantillon d'A. gummifera (J.F. Gmel.) C.A. Sm., a été identifié en comparaison avec celle du collecteur Letouzey 8855 du spécimen de la collection d'herbier à la référence numéro $17050 \mathrm{SRF} / \mathrm{Cam}$.

Description de la recette traditionnelle : Elle est une décoction préparée à partir d'une poignée de main d'adulte de chaque drogue végétale à l'état $\mathrm{sec}$, qui seront par la suite introduites dans une casserole contenant 5 litres d'eau courante et porté à ébullition jusqu'à obtention d'une coloration marron-foncée. En laboratoire, s'inspirant du mode de préparation traditionnelle, la recette est obtenue en mélangeant les différentes poudres ${ }^{2}$ d'écorces de tronc d'Albizia gummifera (A) et Spathodea campanulata (S) dans les proportions respectives de 75/25 (masse/masse) correspondant au rapport masse/masse d'un volume identique de poudre de chacune des drogues végétales mesuré respectivement à l'état sec.

Préparation des extraits végétaux aqueux et hydroéthanoliques : Après récolte, les écorces de tronc de chacune des plantes ont été séchées à la température ambiante à l'ombre durant quatre semaines. Ensuite, à l'aide d'un broyeur électrique (Blender, Warning Commercial, USA), les différentes écorces ont été broyées en poudre fine séparément. Ces différentes poudres ont été utilisées pour la préparation des extraits vers de terre adules. Atsafack et al. ;2015 ont montré que l'extrait brut des écorces d'A. gummifera et ses différentes fractions contenaient des substances dotées de propriétés anti oxydante et antibactérienne in vitro vis-à-vis des espèces du genre Salmonella. Ainsi, considérant l'activité antibactérienne de chacune des plantes prises individuellement, il nous est apparu d'un grand intérêt d'évaluer l'activité antibactérienne qu'aurait la recette traditionnelle associant ces deux espèces végétales.

aqueux et hydro-éthanoliques d'Albizia gummifera (A) et Spathodea campanulata (S), ainsi que ceux de la recette traditionnelle selon la méthode décrite par Duke, 2000.

Les extraits aqueux, représentant le mode d'extraction des tradithérapeutes, ont été obtenus après décoction de cinquante grammes $(50 \mathrm{~g})$ de poudre de chaque plante et mélange de plantes dans les proportions de 75/25 respectivement pour A et $S$ (recette traditionnelle) dans $500 \mathrm{ml}$ d'eau distillée à sa température d'ébullition pendant $15 \mathrm{mn}$. Le macérât obtenu a été refroidi puis filtré deux fois sur du coton hydrophile, puis une fois sur papier Whatman $\mathrm{N}^{\circ} 1$ et le filtrat séché à l'étuve à une température de $45 \pm 1^{\circ} \mathrm{C}$.

- Les extraits hydro-éthanoliques ont été obtenus après macération de cinquante grammes $(50 \mathrm{~g})$ de poudre de chaque plante et mélange de plantes dans les proportions de 75/25 respectivement pour $A$ et $S$ (recette traditionnelle) dans $500 \mathrm{ml}$ du système solvant éthanol / eau $50 / 50$ (v/v) pendant $48 \mathrm{~h}$ sous agitation magnétique à la température ambiante. Les macéras obtenus ont été filtré deux fois sur du coton hydrophile, puis une fois sur papier Whatman $\mathrm{N}^{\circ} 1$ et le filtrat concentré à l'aide d'un évaporateur rotatif (JANKE \& KUNKEL IKA -WERK@) à $65^{\circ} \mathrm{C}$, puis séché à l'étuve à une température de $45 \pm$ $1^{\circ} \mathrm{C}$ pour un séchage complet.

Screening phytochimique : Les grandes classes de composés bioactifs des extraits bruts aqueux et hydroéthanoliques de chaque plante et de la recette ont été recherchées par l'usage des procédures standard tel que décrits par Sofowora, 1993. Les extraits ont été testés qualitativement afin de révéler la présence des grands groupes chimiques comme les tanins, les stéroïdes, les saponosides, les polyphénols, les alcaloïdes et les quinones.

Microorganismes : Deux souches bactériennes, disponibles au Laboratoire de Pharmacognosie et 
Chimie Pharmaceutique de la Faculté de Médecine et des Sciences Biomédicales de l'Université de Yaoundé 1(Cameroun), ont été retenues dans le cadre de cette étude. II s'agissait d'une souche à Gram positive (Staphylococcus aureus ATCC BAA-977) et d'une autre à Gram négative (Escherichia coli ATCC 25922).

Milieux de culture : Deux milieux de culture, préparés selon les recommandations du fabricant, ont été utilisés dans le cadre de cette étude :

- $\quad$ La gélose Mueller Hinton (MH) Liofilchem® pour le repiquage des souches ;

- $\quad$ Le bouillon Mueller Hinton (MHB) Liofilchem® pour la détermination des Concentration Minimales Inhibitrices (CMI).

Préparation des inocula bactériens : Elle a été faite selon les recommandations du Comité de l'Antibiogramme de la Société Française de Microbiologie (CA-SFM, 2015). Pour chacun des deux microorganismes sélectionnés, quelques colonies bactériennes provenant d'une culture pure de 18-24 h sur gélose de Mueller Hinton ont été introduites dans $10 \mathrm{~mL}$ d'eau physiologique de façon à obtenir une opacité équivalente au tube 0,5 de la gamme étalon de

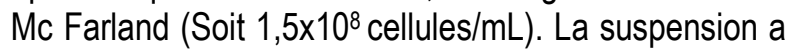

\section{RÉSULTATS}

Caractérisation phytochimique des différents extraits : Le tableau 1 présente les grands groupes de composés chimiques qui ont été caractérisés. II s'agit des alcaloïdes, des coumarines, glycosides, des polyphénols et des saponosides présent dans tous les différents extraits. Les tanins sont présents uniquement dans les extraits aqueux et les Flavonoïdes uniquement dans les extraits aqueux et hydro-éthanolique de $S$. campanulata.

Concentrations Minimales Inhibitrices (CMI) : Elles sont exposées dans le tableau 2. Les différents extraits ont inhibé la croissance de toutes les souches bactriennes testées. Vis-à-vis de Staphylococcus aureus, les CMl étaient comprises entre 3,125 et 12,5 été ensuite diluée avec le MHB au 1/100ème afin d'obtenir un inoculum d'une concentration de $10^{6} \mathrm{UFC} / \mathrm{ml}$.

Préparation des solutions mères d'extraits à tester : Les solutions mères d'extraits aqueux et hydroéthanoliques de chaque plante et de la recette (mélange des deux plantes) ont été préparées à la concentration de $400 \mathrm{mg} / \mathrm{mL}$. A cet effet, pour chaque extrait, $2 \mathrm{~g}$ d'extrait sec ont été introduits dans un tube à essai contenant $5 \mathrm{~mL}$ du système solvant bouillon/DMSO dans des proportions 90/10 V/V. La solution a été homogénéisée au Vortex et ensuite stérilisée à l'autoclave (à $121 \pm 1^{\circ} \mathrm{C}$ durant 15 minutes \pm 1 minute). Détermination de la Concentration Minimale Inhibitrice (CMI) : La Concentration Minimale Inhibitrice (CMI) a été déterminée par la méthode de macro-dilution en milieu liquide avec appréciation visuelle de la croissance des microorganismes tel que préconisé par la Clinical and Laboratory Standards Institute (CLSI, 2012).

Méthodes de saisie et traitement des données : Les données de cette étude portant sur le screening phytochimique et l'activité antibactérienne ont été saisies et les représentations graphiques ont été réalisées dans le tableur EXCEL version 2016.

$\mathrm{mg} / \mathrm{mL}$ pour les extraits aqueux et égales à $0,78 \mathrm{mg} / \mathrm{mL}$ pour les extraits hydro-éthanoliques, vis-à-vis de Escherichia coli les CMI sont comprises entre 6,25 et $200 \mathrm{mg} / \mathrm{mL}$, autant pour les extraits aqueux que pour les extraits hydro-éthanoliques. Les extraits aqueux et hydro-éthanoliques de la recette traditionnelle, bien que présentant une activité antibactérienne vis-à-vis de $S$. aureus avec des valeurs de CMI respectives de 6,25 et $0,78 \mathrm{mg} / \mathrm{mL}$, présente la plus faible activité antibactérienne vis-à-vis de $E$. coli avec des valeurs de $\mathrm{CMl}$ supérieure à $100 \mathrm{mg} / \mathrm{mL}$. Ces résultats nous renseignent sur le fait que la souche de $S$. aureus semble être plus sensible aux différents traitements que la souche de E. coli. 
Bayaga et al., J. Appl. Biosci. 2020 Pouvoir antibactérien des extraits aqueux et hydro-éthanolique du mélange d'écorces de tronc d'Albizia gummifera (J.F. Gmel.) C.A. Sm et Spathodea campanulata P.Beauv

Tableau 1 : Métabolites secondaires présents dans les différents extraits $(A=A$. gummifera et $S=S$. campanulata)

\begin{tabular}{l|l|l|l|l|l|l}
\hline & \multicolumn{3}{|c|}{ Extraits Aqueux } & \multicolumn{3}{c}{ Extraits Hydro-éthanoliques } \\
\hline Métabolites secondaires & A & S & $\begin{array}{l}\text { A+ S } \\
(75 / 25)\end{array}$ & A & S & $\begin{array}{l}\text { A+ S } \\
(75 / 25)\end{array}$ \\
\hline Alcaloïdes & + & + & + & + & + & + \\
\hline Anthocyanes & - & - & - & - & - & - \\
\hline Bétacyanes & + & - & - & + & - & - \\
\hline Coumarines & + & - & + & + & - & + \\
\hline Flavonoïdes & - & + & - & - & + & - \\
\hline Glycosides cardiaques & + & + & + & + & + & + \\
\hline Mucilages & - & - & - & - & - & - \\
\hline Oxalates & - & - & - & - & - & - \\
\hline Phlobotannins & - & - & - & - & - & - \\
\hline Polyphénoles & + & + & + & + & + & + \\
\hline Quinones & - & - & - & - & - & - \\
\hline Résines & - & - & - & - & - & - \\
\hline Saponosides & + & + & + & + & + & + \\
\hline Stéroïdes & - & - & - & - & - & - \\
\hline Tannins & + & + & + & - & - & - \\
\hline
\end{tabular}

Légende:(+) = présence du métabolite recherché et $(-)=$ absence du métabolite recherché.

Tableau 2: CMI des extraits aqueux et hydro-éthanoliques de Spathodea campanulata et Albizia gummifera vis-à-vis de Escherichia coli et Staphylococcus aureus.

\begin{tabular}{l|l|c|c}
\hline \multicolumn{2}{c|}{ Souches bactériennes } & E. coli ATCC 25922 & S. aureus ATCC BAA-977 \\
\hline \multirow{3}{*}{ Extraits aqueux } & A & CMI $(\mathrm{mg} / \mathrm{mL})$ & CMI $(\mathrm{mg} / \mathrm{mL})$ \\
\cline { 2 - 4 } & S & 12,5 & 12,5 \\
\cline { 2 - 4 } & A+S $(75 / 25)$ & 6,25 & 3,125 \\
\hline \multirow{2}{*}{ Extraits hydro-éthanoliques } & A & $>100$ & 6,25 \\
\cline { 2 - 4 } & S & 12,5 & 0,78 \\
\cline { 2 - 4 } & A+S $(75 / 25)$ & 6,25 & 0,78 \\
\hline
\end{tabular}

Légende : $\mathrm{A}=\mathrm{A}$. gummifera et $\mathrm{S}=\mathrm{S}$. campanulata

\section{DISCUSSION}

Screening phytochimique : Les tests phytochimiques réalisés sur les extraits à base d'écorces de tronc de $S$. campanulata montre que les extraits aqueux et hydroéthanolique de ces dernières, contiennent les alcaloïdes, les glycosides, les flavonoïdes les polyphénols, les saponosides et sont exemptes des phobotannins. Des travaux antérieurs réalisés sur les feuilles et fleurs de S. campanulata par Rajesh et al., 2010 et Akharaiyi et al., 2015 ont montré que ces organes étaient également dépourvus de phobotannins et étaient riches en alcaloïdes, glycosides, polyphénols et saponosides. En revanche, d'autres études en l'occurrence celle de Ntie-Kang et al.,2013, révèlent que les quinones, dont le test caractéristique s'est révélé négatif dans la présente étude, constituent la classe de métabolites secondaires la plus représentative de la famille des Bignoniaceae, qui, par ailleurs, est la famille d'appartenance de $S$. campanulata. La recherche infructueuse des stéroïdes et la présence des métabolites secondaires que sont les alcaloïdes, les polyphénols et les saponosides dans les extraits aqueux et hydro éthanoliques d'A. gummifera ont également été observées par Atsafack et al., 2016 lorsqu'il évaluait les propriétés antioxidantes et antibactériennes des extraits et fractions d'extraits des écorces d'Albizia gummifera. Cependant, ces derniers indiquaient la présence des anthocyanes dans les différents extraits des écorces de A. gummifera et dont la recherche s'est révélée négative dans la présente étude. Le screening phytochimique des extraits aqueux et hydro-éthanolique de cette recette a révélé la présence de plusieurs familles de composés chimiques déjà identifiés dans les extraits de chacune 
des plantes entrant dans cette recette à l'instar des alcaloïdes, des glycosides, des polyphénols et des saponosides. Cependant, nous pouvons noter l'absence des tanins dans l'extrait hydro-éthanolique et celle des flavonoïdes dans les extraits aqueux et hydroéthanolique de la recette.

Activité antibactérienne: Les différentes valeurs de Concentrations Minimales Inhibitrices (CMI) obtenues dans la présente étude justifieraient l'inhibition de la croissance de toutes les souches bactériennes testées par les différents extraits. L'usage empirique de chacune de ces plantes séparément et particulièrement en association, dans la prise en charge des infections microbiennes, par les tradithérapeutes à Akonolinga (Centre-Cameroun) se trouve ainsi justifié. L'intensité des effets sur la croissance des bactéries était différente d'une souche à une autre. Desalegn et al., 2014 faisaient un constat similaire lorsqu'ils ont évalué l'activité antibactérienne des extraits de plantes. Selon cet auteur, l'espèce $S$. aureus était moins résistante que $E$. coli. Ceci pourrait se justifier par la complexité de la paroi des bactéries à Gram - par rapport à celle des bactéries

\section{CONCLUSION ET APPLICATION DES RÉSULTATS}

L'étude de l'activité antibactérienne des extraits aqueux et hydro-éthanolique du mélange d'écorces de tronc d'Albizia gummifera et de Spathodea campanulata a été réalisée sur deux souches de référence (Staphylococcus aureus ATCC BAA-977et Escherichia coli ATCC 25922). Les résultats de cette étude suggèrent que les métabolites secondaires en l'occurrence des polyphénoles, flavonoïdes, coumarines, tanins, glycosides cardiaques, bétacyanes, saponosides et alcaloïdes, présents dans ces différents extraits sont

\section{REMERCIEMENTS}

Nous tenons à remercier particulièrement $\mathrm{Mr}$ Ngobo Belinga Rémy, pour nous avoir gracieusement partagé son savoir en nous indiquant cette recette. Nos remerciements vont également à l'endroit du Chef de Département de Pharmacognosie et de Chimie Pharmaceutique de la Faculté de Médecine et des

\section{BIBLIOGRAPHIE}

Adjet AA, Kouame D, Fokou G, 2016. Phytothérapie et lutte contre l'ulcère de Buruli dans le district sanitaire de Yamoussoukro (Cote d'lvoire) : identification, description, fonction symbolique des plantes et recettes utilisées. Médecine et Santé Tropicales. 26 : 408-413. Doi : 10.1684/mst.2016.0630. à Gram + ; mais également par le mécanisme d'action des différents extraits bien qu'il n'est pas étudié dans cette étude. De nombreuses études ont mis en évidence l'activité antibactérienne des extraits de chacune de ces deux plantes (Albizia gummifera et $S$. campanulata) visà-vis de nombreux microorganismes responsables des infections à l'instar de Yemoa et al., 2011 sur Mycobactérium ulcerans ; Sowjanya et al., 2013 sur de nombreuses souches de bactériennes et fongiques ; Gebregergs et al., 2015 et Atsafack et al., 2016 sur des souches du genre Salmonella. Ces auteurs ont attribué cette activité à la présence, dans les différents extraits, de métabolites secondaires en l'occurrence des tannins, des flavonoïdes, des polyphénols, des glycosides et des alcaloïdes qui, pour la plupart, sont également présents dans les différents extraits testés dans la présente étude à l'exception des flavonoïdes qui sont absents dans les extraits aqueux et hydro-éthanolique d'A. gummifera et celle de la recette; ce qui justifierait la différence des valeurs de CMI obtenues avec ces extraits et ceux obtenues avec les extraits de $S$. campannulata.

responsables de l'activité antibactérienne observée sur les bactéries utilisées. Ces résultats montrent également que l'intensité de l'inhibition de la croissance visible est variable d'une souche à l'autre. Ce résultat pourrait justifier l'usage traditionnelle de cette recette vis-à-vis des infections à Staphylococcus aureus bien que des études complémentaires soient nécessaires pour déterminer la nature de l'interaction pouvant exister entre les constituants de cette recette.

Sciences Biomédicales de l'Université de Yaoundé I, ainsi qu'à toute l'équipe du dit département pour l'assistance technique et matériels indispensables à la réalisation de ce travail. Nous remercions les relecteurs qui ont contribué à améliorer la qualité de cet article.

Akharaiyi FC, Boboye B, Akpambang VO, Adetuyi FC, 2015. Phytochemical and Antioxidant Effect of Spathodea campanulata leaf Extracts. International Journal of Biochemistry Research \& Review. 7(3): 148-59.

Atsafack SS, Kodjio N, Chegaing Fodouop SP, Ntemafack A, Kuiate JR, Gatsing D, 2016. In 
vitro Antisalmonellal and Antioxidant Activities of the Crude Extracts and Fractions from the Stem Bark of Albiziagummifera (J. F. Gmel.) C. A. Sm. British Journal of Pharmaceutical Research. $10(6): 1-11$.

Bayaga HN, Guedje NM et Biye EH, 2017. Approche ethnobotanique et ethnopharmacologique des plantes utilisées dans le traitement traditionnel de l'ulcère de Buruli à Akonolinga (Cameroun). International. Journal of Biological and Chemical Sciences. 11(4): 1523-1541.

Clinical and Laboratory Standards Institute (CLSI),2012. Methods for Dilution Antimicrobial Susceptibility Tests for Bacteria That Grow Aerobically; Approved Standard-Ninth Edition. 32(2) :88p.

Comité de l'antibiogramme de la société française de microbiologie (CA-SFM),

2015. Recommandations.117p.

Desalegn A, 2014. Antimicrobial Activity of Medicinal Plant Extracts and Their Synergistic Effect on Some Selected Pathogens.American Journal of Ethnomedicine. 1(1): 18-29.

Duke JA, 2000. Le Pouvoir des Plantes. Encyclopédie des Plantes Médicinales du Département de Phytothérapie de Bobigny. France $2{ }^{\text {ème }}$ edition. 620p. ISBN: 2-501-03356-6.

Gebregergs T, Biniam T, Tadesse E, Alehegn W, 2015. In vitro Screening of Antibacterial Activities of Selected Ethiopian Medicinal Plants. International Journal of Microbiological Research. 6(1) :27-33.

Haut conseil de la santé publique commission spécialisée Maladies transmissibles, 2011. Les maladies infectieuses émergentes : état de la situation et perspective.207p.

Ntie-Kang F, LikowoLifongo L, Meva'aMbaze L, NnangeEkwelle, Owono LC, Megnassan E, Judson PN, Wolfgang S, Efange SMN, 2013. Cameroonian medicinal plants: a bioactivity versus ethnobotanical survey and chemotaxonomic classification. BMC Complementary and Alternative Medicine. 13(147): :18p.

Organisation Mondiale de la Santé(OMS), 2013. Stratégie pour la médecine traditionnelle 20142023. Genève. 72p.

Pone Kamdem Boniface, 2017. "Advances on Ethnomedicinal Uses, Phytochemistry, and Pharmacology of Spathodea campanulata P. Beauv". EC Pharmacology and Toxicology. 5(2): $51-62$
Rajesh Kowti, Harsha R, Mohammed GA, Hareesh AR, Thammanna Gowda SS, Dinesha R, Satish Kumar BP, Irfan Ali M, 2010. Antimicrobial activity of leaf and flower of Spathodea campanulata P. Beauv. Research Journal of Pharmaceutical, Biological and Chemical Sciences. 1(3):8.

Sofowora A.,1993. Medicinal plants and traditional medicine in Africa. John Wiley and sons LTD. 97-145.

Sowjanya PR, Kiran B, Srinivasa BP,2013. The efficacy of spathodea campanulata P. Beauv leaf extract on some bacterial and fungal strains by well diffusion method. 4(3):909-916.

Tanayen JK, Ajayi AM, Oloro J, Ezeonwumelu JOC, Tanayen GG, Adzu B,Arthur van Aerschot, Gert L, Agaba AG, 2016. Phytochemical and Antidiabetic Evaluation of the Methanolic Stem Bark Extract of Spathodea campanulata ( $P$. Beauv.) Bignoniaceae. Pharmacognosy Journal. 8 :243-249. DOI: 10.5530/pj.2016.3.12.

Traoré Y, Ouattara K, Yéo D, Doumbia I, Coulibaly A, 2012. Recherche des activités antifongique et antibactérienne des feuilles d'Annona senegalensis Pers. (Annonaceae). Journal of Applied Biosciences. 58 :4234- 4242.

Wagh AS \& Butle SR, 2018. Plant profile, phytochemistry and pharmacology of Spathodea campanulata $P$. Beauvais (african tulip tree): a review. International Journal of Pharmacy and Pharmaceutical Sciences.10 (5):1-6

Wagh A, Butle S, Telang $p, 2019$. In Vitro Anthelmintic Efficacy of Spathodea campanulata P. Beauv. (Bignoniaceae) Against Pheretima posthuma. Asian Journal of Pharmacognosy. 3(1):32-38.

Yemoa AL, Gbenou J, Affolabi Di, Moudachirou M, Bigot A, Anagonou S, 2011. Buruli Ulcer: A Review of in Vitro Tests to Screen Natural Products for Activity against Mycobacterium ulcerans. Planta Med. 77 : 641-6. 